Results: Out of 59 bone marrow biopsy reports reviewed, four $(\sim 7 \%)$ cases did not describe morphological features consistent with the stated diagnoses based on WHO diagnostic criteria. Quantitative assessment of reported dysplasia $(>/<10 \%)$ was infrequently provided, with qualitative terms more commonly used. Fifty-three reports complied with RCPA structured reporting guidelines, but six $(\sim 11 \%)$ omitted details regarding trephine length, iron stores or presence and proportion of ring sideroblasts. WHO disease subtype was provided in all cases, but ICD-O code was rarely included $(\sim 8 \%)$.

Discussion: Reporting of morphological features in MDS according to WHO criteria in a structured format based on published guidelines allows objective confirmation and standardisation of MDS diagnoses, and identifies areas for improvement.

References

1. Swerdlow SH, Campo E, Harris NL, et al., editors. WHO Classification of Tumours of Haematopoietic and Lymphoid Tissues. Revised 4th ed, 2016. Lyon: IARC, 2017.

2. Royal College of Pathologists of Australasia (RCPA). Bone Marrow Specimen (Aspirate and Trephine Biopsy) Structured Reporting Protocol (1st Edition 2014). Sydney: RCPA, 2014. https://www.rcpa.edu.au/Library/Practising-Pathology/

Structured-Pathology-Reporting-of-Cancer/Cancer-Protocols

\section{DOAC STOP USE IN REAL LIFE - A SINGLE CENTRE VALIDATION STUDY}

Sean McKeague, Robyn Coleman, Rebecca Adams, Timothy Stanton, Tee Beng Keng, Yasmin Harvey Department of Haematology, Sullivan Nicolaides Pathology, Brisbane, Qld, Australia

Background: DOAC STOP (Haematex Research, Sydney) is an activated charcoal based agent that absorbs all commonly used direct oral anticoagulants (DOACS) from plasma, enabling assessment of underlying coagulation. ${ }^{1}$ There is limited data on the effect of DOAC STOP on less common factor assays and on true positive lupus anticoagulant (LA) tests. ${ }^{2}$

Method: A local validation study of DOAC STOP was undertaken at Sullivan Nicolaides central laboratory (Bowen Hills, Queensland). Efficacy in eliminating dabigatran and rivaroxaban was assessed by comparing anti-Xa assays and TCT pre and post $\operatorname{DOAC~STOP}(n=7)$. Common coagulation assays, extended factor levels and prothrombotic screen were tested pre and post DOAC stop in normal samples $(n=43)$. LA testing was performed pre and post DOAC STOP in patients with known LA positivity $(n=10)$. Results: DOAC stop effectively removed dabigatran and rivaroxaban. There was no major difference $(>10 \%)$ in any of the assays pre and post DOAC stop. All 10 samples with detectable LA remained detectable after treatment with DOAC STOP.

Discussion: This is the first study reporting the stability of less common factor assays with DOAC STOP. Results suggest that DOAC STOP does not abolish detected LA results, therefore providing a valid method for detecting LA whilst on DOAC therapy. ${ }^{3}$

\section{References}

1. Exner T, Michalopoulos N, Pearce J, et al. Simple method for removing DOACs from plasma samples. Thromb Res 2018; 163: 117-22.

2. De Kesel PM, Devreese KMJ. Direct oral anticoagulant adsorption: impact on lupus anticoagulant testing-Review of the literature and evaluation on spiked and patient samples. $J$ Thromb Haemost 2020; 18: 2003-17.

3. Favaloro EJ, Gilmore G, Arunachalam S, et al. Neutralising rivaroxaban induced interference in laboratory testing for lupus anticoagulant (LA): a comparative study using DOAC Stop and andexanet alfa. Thromb Res 2019; 180: 10-19.

\section{USE OF MULTIDIMENTIONAL FLOW CYTOMETRY PLOTS TO IMPROVE MEASURABLE RESIDUAL DISEASE MONITORING OF ACUTE MYELOID LEUKAEMIAS}

Riana van der Linde $^{1}$, Sandy Smith ${ }^{2}$, Sarah C. Sasson ${ }^{2,3,4}$, David Brown ${ }^{2,3,4}$, Elizabeth Tegg ${ }^{1,2,4}$

${ }^{1}$ Department of Laboratory Haematology, ICPMR, Westmead Hospital, NSW Health Pathology, NSW, Australia; ${ }^{2}$ Flow Cytometry Unit, Department of Immunopathology, ICPMR, NSW Health Pathology, NSW, Australia; ${ }^{3}$ Department of Clinical Immunology, Westmead Hospital, NSW, Australia; and ${ }^{4}$ Sydney Medical School, Faculty of Medicine and Health, University of Sydney, NSW, Australia

Background: Acute myeloid leukaemia (AML) is a haematological malignancy with a prevalence of $1.5 \%$. ${ }^{1}$ Improved AML management requires increasingly sensitive measurable residual disease (MRD) detection, by either molecular or flow cytometric methods. ${ }^{2}$ We hypothesised that the effect of phenotypic changes, a well-known feature of AML, on multiparameter flow cytometry MRD (MFC-MRD) analysis can be minimised by using multidimensional radar plots.

Methods: We created a novel MRD analysis template using multidimensional radar plots (Kaluza software). The analysis method was validated with 80 consecutive MFC-MRD samples, followed by a testing cohort $(n=36)$.

Results: The two MRD methods showed a moderate correlation $(k=45 \%)$ with no statistically significant difference $(p=0.21)$. Nineteen tests were positive using the radar plot method and negative with our current method. 14/19 (74\%) had low-level MRD, between $0.01 \%$ and $0.1 \%$. Molecular results were available for $36 / 116$ cases only showing a slight correlation $(k=15 \% ; p=1.0)$. Discussion: Moderate agreement between the two MFC methods suggest the radar plot method is fit for purpose. Discrepancies can partially be explained by increased sensitivity of molecular tests. Other possible confounding factors include leukaemic subclones and treatment-related phenotypic changes. ${ }^{3}$ We are currently conducting a prospective MRD study to better understand these findings.

\section{References}

1. World Health Organization. 2014 Review of Cancer Medicines on the WHO List of Essential Medicines. Cited 1 Apr 2021. http://www.who.int/selection_medicines/committees/ expert/20/applications/AML_APL.pdf.

2. Zeijlemaker W, Gratama JW, Schuurhuis GJ. Tumor heterogeneity makes AML a "moving target" for detection of residual disease. Cytometry B Clin Cytom 2014; 86: 3-14.

3. Li W, Morgan R, Nieder R, et al. Normal or reactive minor cell populations in bone marrow and peripheral blood mimic minimal residual leukemia by flow cytometry. Cytometry $B$ Clin Cytom 2020; Nov 16: 10.1002/cyto.b.21968. 